\title{
CONSIDERACIONES DE DIRECTORES DE LOS CENTROS DE EDUCACIÓN INFANTIL Y PRIMARIA DE LA COMUNIDAD AUTÓNOMA DE VALENCIA RESPECTO A DIVERSOS ASPECTOS DE SU CARGO
}

\author{
OPINIONS ON THEIR POSITIONS BY PRE-SCHOOL AND PRIMARY \\ SCHOOL HEADTEACHERS IN THE AUTONOMUS REGION OF \\ VALENCIA REGARDING VARIOUS ASPECTS OF THEIR CHARGE
}

María González-Álvarez ${ }^{a^{*}}$

Fechas de recepción y aceptación: 30 de junio de 2018, 11 de junio de 2019

Resumen: En el presente estudio analizamos las valoraciones que directores y directoras de centros públicos, privado-concertados y privados de Educación Infantil y Primaria de la comunidad autónoma de Valencia realizan en relación con diversos aspectos de su formación y funciones. Una prueba elaborada por la autora ha sido cumplimentada por 71 responsables de la dirección. Estos directivos consideran que la formación inicial recibida en sus estudios para profesorado, respecto a su actual ejercicio profesional, no ha sido la adecuada. Valoran como mejorable la formación permanente que reciben de las administraciones respecto a dichas responsabilidades. Formación que debe ir orientada a temas como la normativa, el liderazgo, la organización y la evaluación de centros, la pedagogía-psicología y las nuevas tecnologías. Están bastante satisfechos en el ejercicio de sus tareas y consideran que su cargo es importante para el funcionamiento del centro y la calidad de la enseñanza que este ofrece. Reciben en su trabajo un razonable apoyo y colaboración por parte de las administraciones. Consideran que su trabajo está bien reconocido por el profesorado y las familias. Están de acuerdo en que debe existir una auténtica carrera profesional para los docentes. Las funciones y competencias que tienen asignadas las consideran importantes para el funcionamiento del centro y la calidad de la enseñanza. Entendemos que las administraciones educativas deben prestar un mejor y mayor reconocimiento a los directores y directoras de los centros de

a Departamento Formación y Orientación Laboral. IES “La Quintana” (Ciaño-Langreo). Consejería de Educación del Principado de Asturias.

* Correspondencia: Formación y Orientación Laboral. IES "La Quintana” (Ciaño-Langreo). Calle Jaime Alberti s/n. 33900 Ciaño-Langreo. Asturias. España.

E-mail: maria.gz.az@gmail.com 
Educación Infantil y Primaria públicos y privado-concertados y de manera especial respecto a la retribución económica que deben percibir por su específico trabajo.

Palabras clave: dirección escolar, formación inicial, formación permanente, Educación Infantil, Educación Primaria, funciones dirección.

Abstract: In this paper we aim to analyze the assessments of Pre-school and Primary Education headteachers in the autonomous region of Valencia in relation to several aspects of their training and responsibilities. A test prepared by the author has been answered by 71 headmasters. They consider that the initial training provided when studying their degrees in education has not been satisfactory, taking in consideration their present professional duties. They think that the ongoing training provided by the educational administration can be improved in relation to these duties. The training should be focused on topics such as regulation, leadership, organization and assesment of schools, pedagogy-psychology and new technologies. They are quite satisfied in the performance of their duties and they consider that their position is important for the management of the school and the quality of the education. They feel they receive a reasonable support, collaboration and consideration on the part of the administration. They believe that their work is well considered by teachers and families. They agree on the fact that there must be a real professional promotion for teachers. The responsabilities and competences assigned are considered important for the running of the school and the quality of the education. We consider that the educational administration should improve the conditions of the head teachers in Public, subsidized and private Elementary and Primary school, specially in relation to the remuneration of their specific responsabilities.

Keywords: school management, initial training, permanent education, Children's Education, Primary Education, director functions.

\section{INTRODUCCIÓN}

La función directiva de los centros educativos se considera un factor fundamental para su buen funcionamiento y la mejora de la enseñanza de su alumnado (Branch, Hanushek y Rivkin, 2012; Fernández-Serra, 2002; Gairín y Castro, 2010; Mulford, 2006; Ritacco y Bolívar, 2016; Vázquez, Liesa, y Gayán, 2013; Waters, Marzano y McNulty, 2003). Función directiva que ante las actuales demandas sociales, conlleva importantes tareas de difícil desempeño, generando incertidumbre en la decisión para asumirla e inestabilidad en su ejercicio (Álvarez-Fernández, 2004; Gairín y Castro, 2010; MartínMoreno, 2007).

Con objeto de conseguir un mejor funcionamiento de los centros y la consiguiente mejora de la enseñanza del alumnado, analizamos las opiniones que 
directores y directoras de los centros públicos, privado-concertados y privados de educación infantil y primaria de la comunidad autónoma de Valencia tienen en relación con diversos aspectos de sus funciones. Tenemos en cuenta las valoraciones que realizan sobre la formación inicial y permanente que han recibido y reciben respecto a sus tareas, así como la importancia que según su criterio tiene su cargo directivo para el buen funcionamiento de los centros y la calidad de la enseñanza que ofrecen a su alumnado. Estudiamos el importante aspecto de la satisfacción que tienen en su trabajo y la valoración que realizan de las retribuciones que perciben por él. Consideramos el análisis que realizan respecto a la colaboración y el reconocimiento que reciben por parte de las administraciones educativas, profesorado y familias. Aspecto de gran interés es estudiar las valoraciones que dichos directores y directoras otorgan a las funciones y competencias que tienen asignadas en relación con el funcionamiento de los centros y la calidad de la enseñanza que ofrecen.

Respecto a la formación inicial y permanente de directores y directoras, Fernández-Serra (2002) señala que debe ser un proceso necesario antes del nombramiento del responsable de la dirección y que ha de desarrollarse a lo largo de toda su etapa directiva. González-Álvarez (2017a, 2017b) analiza, en un estudio con directivos de todas las comunidades de España, tanto la formación respecto a la función directiva recibida en los estudios previos al ejercicio profesional como la que las administraciones educativas les ofrecen en sus habituales tareas directivas. En el primer caso, se entiende que dicha formación inicial debe mejorarse sustancialmente, y en lo que respecta a la formación permanente deberían implementarse programas relacionados con la normativa, liderazgo, organización y evaluación de centros, pedagogía-psicología y nuevas tecnologías, entre otros. Según Aramendi, Teixidó y Bernal (2010), para el 95,2 \% de directores y directoras la formación respecto a la gestión directiva debe incluirse en los currículos universitarios de formación del profesorado. En relación con los programas de formación de los directivos en educación, Gómez-Delgado, Oliva-Rodríguez y López-Yáñez (2014) señalan que los de formación inicial prestan mayor atención a la gestión de centros, legislación y organización, mientras que en la formación continua los contenidos se refieren a las relaciones interpersonales, que facilitan una dirección más participativa, y al liderazgo pedagógico centrado en la mejora de los resultados del aprendizaje. También respecto a la formación, Vázquez, 
Liesa y Gayán (2013) entienden que no solo no se responde en sus programas al modelo de dirección sino que tampoco se hace en relación con las necesidades sentidas por directores y directoras. Para Hess y Kelly (2007) la formación previa al servicio es deficiente en temas clave de gestión, como el manejo de personal y el mantenimiento de las instalaciones. La función formativa de los propios establecimientos educativos es para Braslavsky (2006) un importante instrumento de los directivos para lograr unas escuelas de calidad. Según el Educobarómetro (2010), los ámbitos en que los directores y directoras se sienten peor preparados, en relación con su formación para ejercer las funciones que tienen asignadas, son los de dirección y evaluación y reclaman más formación en legislación educativa y nuevas tecnologías.

Por otro lado, el liderazgo, que se considera hoy vinculado a la función directiva, debe tener una importante vertiente pedagógica (Calatayud, 2015; García-Garnica, 2016), a la cual se le restan posibilidades por la excesiva gestión administrativista (Bolívar y Cerrillo, 2015). Un liderazgo que, a su vez, ha de estar distribuido entre diferentes miembros de la comunidad escolar (García-Garnica, 2016). En relación con la formación recibida respecto al liderazgo, se valora como insuficiente por el $41,8 \%$ de las personas consultadas en el estudio realizado por Vázquez, Liesa y Bernal (2015).

Respecto al importante tema del modelo de selección de los responsables de la dirección de los centros públicos en España, señala Batanaz (2005), en su trabajo de hace algo más de una década, que en el ámbito de la investigación nos encontramos con dos grupos, uno en el que se defiende un modelo de selección del director o directora con participación de representantes de los distintos miembros de la comunidad escolar del centro, y teniendo en cuenta los méritos profesionales de los candidatos, con sus necesarias modificaciones, y otro donde están los que se hallan a favor de la profesionalización de la función directiva. Por otro lado, Bolívar y Ritacco (2017), en un reciente trabajo, indican que en el modelo de dirección escolar español el director debe ejercer un doble rol (docente y director-docente), así como ocupar un lugar intermedio entre administración y profesorado, con la problemática que ello conlleva.

Respecto a la satisfacción en la responsabilidad del director, en el informe TALIS (2009) se señala que en el conjunto de los países y regiones de la OCDE el 95,5\% de los directores manifiestan estar satisfechos con su traba- 
jo. En el estudio llevado a cabo por Caballero y Mata (2004), el 4,6 \% de los responsables de la dirección de los centros tienen un grado de insatisfacción general alta o moderada; algo más de la mitad (53,8 \%) se muestran satisfechos o muy satisfechos, y el 31,5 \%, ni satisfechos ni insatisfechos. Los directores escolares muestran altos niveles de satisfacción, de forma jerarquizada; según Tejero y Fernández (2009), en primer lugar con el equipo directivo y el personal de secretaría, en segundo lugar con el profesorado, el alumnado y las familias, y finalmente con las condiciones laborales. Tellez (2015) considera que el profesorado de Educación Infantil y Primaria tiene un nivel de satisfacción alto en su relación profesional con el director o directora y con sus colegas docentes.

Según García, Poblete y Villa (2006), las funciones desempeñadas por los cargos directivos se consideran importantes, y realizadas las relativas a la información, planificación, coordinación, representación y gestión de recursos, siendo valoradas como importantes pero poco realizadas las funciones de control-evaluación, animación pedagógica y dirección, cuando supone un ejercicio de autoridad. Grissom y Loeb (2011) estudian la eficacia de los directivos y señalan que se sienten más eficaces para desarrollar relaciones con los estudiantes, comunicarse con los padres, asistir a actividades escolares, generar entornos escolares seguros, atender las inquietudes del personal, administrar los horarios escolares y usar los datos para informar la instrucción. Los directivos se consideran menos eficaces respecto al asesoramiento a los maestros, utilizar las comunicaciones de la oficina del distrito para mejorar sus metas y trabajar con miembros de la comunidad local y organizaciones.

Al analizar las actuaciones de los directores y directoras, según el modelo de dirección autopercibido, Barrios, Camarero, Tierno-García e IranzoGarcía (2015) resaltan el alto grado de realización (95\% de los directores), considerando que son actuaciones señaladas en la normativa. Por otro lado, al estudiar el desarrollo de dichas actuaciones en función del modelo de dirección, se observa que coinciden en la realización de las actividades referidas a presidir los órganos de gobierno y actos académicos, en garantizar los derechos y deberes de los miembros de la comunidad educativa y en proponer la programación anual del centro.

Respecto al uso del tiempo por parte de los directores y directoras, según Murillo y Hernández-Castilla (2015), estos dedican el 36,4 \% de su tiempo 
a tareas relativas a la administración y organización de su planificación, el $26,7 \%$ a las de liderazgo pedagógico, el 15,6 \% a las relaciones con las familias, el $14,95 \%$ al desarrollo personal y el $6,31 \%$ a otras tareas.

\section{MÉTodo}

\subsection{Muestra}

Hemos consultado a directores y directoras de los centros públicos, privado-concertados y privados de Educación Infantil y Primaria de la comunidad autónoma de Valencia para conocer sus opiniones sobre su formación inicial y permanente como director o directora, su satisfacción y reconocimiento en su cargo, la importancia de la dirección para el buen funcionamiento del centro y la calidad de la enseñanza que ofrece el centro, carrera profesional, retribución económica y colaboración que reciben en sus funciones por parte de los restantes miembros de la comunidad escolar.

Para realizar un análisis exploratorio hemos elaborado un protocolo que ha sido enviado por correo electrónico a una muestra de centros seleccionados al azar y en función de la población de las distintas provincias de la comunidad y de la titularidad de los centros. La muestra seleccionada ha sido extraída del Registro Estatal de Centros Docentes no Universitarios del Ministerio de Educación. Entendemos que el procedimiento online empleado gana en cantidad y en muestreo al azar frente a otras opciones, a la vez que puede ser el procedimiento adecuado no solo por la técnica utilizada con la aplicación de Mailchimp, sino por su facilidad para emitir y recoger las respuestas con la aplicación de Google. Según Escudero-Muñoz, González-González y Rodríguez-Entrena (2018), este tipo de instrumento se utiliza frecuentemente en la investigación sobre formación docente. Encontramos como importante inconveniente la desconfianza que genera la recepción de un correo electrónico no identificado y su consiguiente eliminación sin llegar a conocer su contenido, lo cual reduce ostensiblemente el número de posibles respuestas recibidas. Al cuestionario le acompañaba una carta en la que señalábamos los objetivos del trabajo a la vez que se agradecía la colaboración del director o directora y se informaba del tratamiento confidencial de la información. La 
muestra elegida es de un tercio del total de los centros indicados en el registro señalado para toda la comunidad (457 centros), teniendo en cuenta que han abierto el correo el $24,5 \%$ de los enviados, es decir, 112, según se indica en la correspondiente aplicación de Mailchimp.

\subsection{Variables e instrumentos}

El objetivo fundamental de nuestro trabajo es realizar un estudio exploratorio que nos informe sobre la actual situación de la función directiva en los centros públicos, privado-concertados y privados que imparten enseñanzas de Educación Infantil y Primaria de la comunidad autónoma de Valencia.

El protocolo puesto a disposición de directores y directoras indicado para su valoración consta de los elementos siguientes: 1. Variables de clasificación: 1.1. Género. 1.2. Edad. 1.3. Tiempo de experiencia profesional. 1.4. Tiempo de experiencia en la función directiva. 1.5. Titularidad del centro. 1.6. Tamaño del centro. 2. Formación inicial y permanente: 2.1. Formación inicial respecto a la gestión directiva recibida en sus estudios previos a la actividad profesional. 2.2. Formación que reciben los actuales estudiantes de profesorado respecto a la gestión directiva. 2.3. Formación permanente que reciben directores y directoras por parte de las administraciones educativas. 3. La formación permanente de los responsables de la dirección de los centros respecto a: 3.1. Normativa. 3.2. Liderazgo. 3.3. Organización de centros. 3.4. Pedagogía-psicología. 3.5. Evaluación de centros. 3.6. Nuevas tecnologías. 4. Satisfacción y reconocimiento del cargo de director o directora: 4.1. Satisfacción en su cargo de director o directora en el centro. 4.2. Importancia de la dirección para el buen funcionamiento del centro. 4.3. Importancia que tiene el director o la directora para la calidad de la enseñanza que ofrece el centro. 4.4. Apoyo y colaboración de las administraciones educativas a la dirección del centro. 4.5. Reconocimiento del trabajo del director o directora del centro por parte de las administraciones educativas. 4.6. Reconocimiento del trabajo de la dirección por parte del profesorado del centro. 4.7. Reconocimiento del trabajo del director o directora por parte de las familias del centro. 5. Carrera profesional, retribución económica y colaboración con la función directiva: 5.1. Existencia de una auténtica carre- 
ra profesional de los docentes. 5.2. Valoración de la retribución económica por el desempeño de las funciones de director o directora del centro. 5.3. Colaboración del profesorado con la dirección del centro. 5.4. Colaboración del profesorado con el centro en general. 5.5. Colaboración de las familias con el centro en general. 6. Importancia de las funciones y competencias de la dirección para el funcionamiento de los centros y la calidad de la enseñanza que ofrecen: 6.1. Ostentar la representación del centro y representar a la Administración educativa en este. 6.2. Ejercer la dirección pedagógica y promover la innovación educativa. 6.3. Garantizar el cumplimiento de las leyes y demás disposiciones vigentes y ejercer la jefatura de todo el personal adscrito al centro. 6.4. Favorecer la convivencia en el centro e impulsar la colaboración con las familias. 6.5. Fomentar un clima escolar que favorezca el estudio y el desarrollo de cuantas actuaciones propicien una formación integral en conocimientos y valores de los alumnos y alumnas. 6.6. Impulsar las evaluaciones internas del centro y colaborar en las evaluaciones externas y en la evaluación del profesorado. 6.7. Convocar y presidir los actos académicos y las sesiones del Consejo Escolar y del Claustro del profesorado del centro, así como ejecutar los acuerdos adoptados por los órganos colegiados, en el ámbito de sus competencias. 6.8. Realizar las contrataciones de obras, servicios y suministros, así como autorizar los gastos de acuerdo con el presupuesto del centro. 6.9. Proponer a la Administración educativa el nombramiento y cese de los miembros del equipo directivo, en su caso. 6.10. Aprobar la programación general anual del centro, sin perjuicio de las competencias del Claustro del profesorado, en relación con la planificación y organización docente. 6.11. Impulsar la colaboración con instituciones y con organismos que faciliten la relación del centro con el entorno, así como fijar las directrices para la colaboración, con fines educativos y culturales, con las administraciones locales, con otros centros, entidades y organismos.

Respondieron al protocolo propuesto 71 responsables, es decir, el 63,4 \% de los que habían abierto el correo, de los centros públicos, privado-concertados y privados que imparten enseñanzas de Educación Infantil y Primaria de la comunidad autónoma de Valencia, siendo el 53,5\% directoras y el 46,5\% directores. Su media de edad es de 48,1 años (directoras 47,2 años y directores 49,1 años). La experiencia profesional media es de 22,8 años (directoras 
21,6 y directores 24,1), y en la dirección de centros 7,2 años (directoras 6,6 y directores 8,0 ). Según la titularidad de su centro el 74,6 \% son de centros públicos, el 23,9\% privados concertados y el 1,5\% privados.

Realizamos los distintos análisis estadísticos mediante descriptivos, diferencia de medias, análisis multivariado (utilizando el método Scheffe) y las pruebas no paramétricas de la U de Mann-Whitney.

Los 72 centros que dirigen los directores y las directoras que responden a las cuestiones planteadas están constituidos por 1.144 unidades, 25.960 alumnos y alumnas y 1.995 profesores y profesoras.

La validez de contenido de dicha prueba la hemos realizado mediante "juicio de expertos" (Escobar-Pérez y Cuervo-Martínez, 2008). A tal fin se ha contado con la colaboración de tres inspectores de educación y siete directores y directoras de centros de Educación Infantil y Primaria, de los cuales cinco gestionan centros públicos y dos privado-concertados. De manera individual se les ha presentado a cada uno de los citados expertos y expertas un protocolo en el que se indicaba el objetivo fundamental del trabajo, extracción de la muestra, aspectos que analizar de la formación de directores y directoras, competencias directivas establecidas en el artículo 132 de la Ley Orgánica de 2/2006, de 3 de mayo de educación, y en el artículo 54.2 de la Ley Orgánica 8/1985, de 3 de julio, reguladora del derecho a la educación, satisfacción y reconocimiento del cargo de director o directora, colaboración con la función directiva y retribución económica. Las aportaciones fundamentales que han realizado los citados expertos y expertas al documento inicial se refieren a los temas relativos a la colaboración de profesorado y familias con el centro en general (como contrapunto a la referida a la colaboración con el director o directora), la formación inicial en sus estudios universitarios y la existencia de una auténtica carrera profesional de los docentes. Han entendido, asimismo, que son adecuadas las variables de clasificación analizadas, así como los aspectos de la formación de directores y directoras propuestos y las competencias directivas que se analizan de acuerdo con el artículo 132 de la Ley Orgánica de 2/2006, de 3 de mayo, de educación, y el artículo 54.2 de la Ley Orgánica 8/1985, de 3 de julio, reguladora del derecho a la educación.

Respecto a la validez del constructo podemos señalar que la medida de adecuación muestral de Kaiser-Meyer-Olkin (KMO) tiene un valor de ,868 
y la prueba de esfericidad de Bartlett tiene un grado de significación de ,000. En el análisis factorial el método de extracción utilizado ha sido el Análisis de Componentes Principales y el método de rotación Varimax con Kaiser. La rotación ha convergido en coho iteraciones. Siete factores explican el 60,6 \% de la varianza.

El protocolo de recogida de información consta de 38 ítems, de los cuales seis son variables de clasificación. Los 32 restantes se distribuyen de la siguiente manera, con el coeficiente de fiabilidad de Cronbach correspondiente: tres elementos de la valoración inicial y permanente tienen un coeficiente de Cronbach de 0,72. El de los temas de formación permanente, 0,86. Dos elementos sobre la importancia del director y directora en el funcionamiento del centro y en la enseñanza del alumnado, 0,73. Los once elementos de la valoración de las competencias del director y directora para el buen funcionamiento del centro y la enseñanza que estos ofrecen tienen un coeficiente de fiabilidad de 0,83 . Satisfacción en el cargo, reconocimiento y colaboración, carrera profesional, retribución económica y méritos, en nueve elementos, tiene un coeficiente de Cronbach de 0,80. La fiabilidad total de la prueba, según el coeficiente de Cronbach, es de un valor de Alpha $=0,87$ (en la escala de 0 a 1), en el total de los 32 elementos analizados.

TABLA 1

Coeficientes de fiabilidad de Cronbach

\begin{tabular}{|l|c|}
\hline \multicolumn{1}{|c|}{ Temas } & $\begin{array}{c}\text { Coeficiente de } \\
\text { Cronbach }\end{array}$ \\
\hline 1. Valoración de la formación inicial y permanente (3 elementos) &, 72 \\
\hline 2. Temas de formación permanente (6 elementos) &, 86 \\
\hline $\begin{array}{l}\text { 3. Importancia del director y directora en el funcionamiento del centro y en la } \\
\text { enseñanza del alumnado (2 elementos) }\end{array}$ &, 73 \\
\hline 4. Importancia de las competencias del director y directora (11 elementos) &, 83 \\
\hline $\begin{array}{l}\text { 5. Satisfacción en el cargo, reconocimiento y colaboración, carrera profesional, } \\
\text { retribución económica, méritos (10 elementos) }\end{array}$ &, 80 \\
\hline Total (32 elementos) &, 87 \\
\hline
\end{tabular}




\section{RESULTADOS}

\subsection{Formación inicial y permanente}

Los responsables de la dirección valoran con 3,4 puntos (escala de 0 a 10 puntos, siendo 0 "nula o nada adecuada" y 10 "muy adecuada") la formación inicial que han recibido, respecto a la gestión directiva, en sus estudios previos a la actividad profesional. Con 3,3 puntos la formación que reciben, en relación con dicha función, los actuales aspirantes a profesorado. Y la formación permanente que les proporcionan las administraciones educativas en el ejercicio de sus funciones directivas la valoran con 4,8 puntos.

Se encuentran diferencias respecto a la valoración de la formación permanente según la titularidad; valoran mejor dicha formación directores y directoras de centros públicos que los de centros privados concertados (centros públicos: 5,3 puntos; privados concertados: 3,4; grado de significación: ,007).

TABLA 2

Formación inicial y permanente

\begin{tabular}{|l|c|c|}
\hline \multicolumn{3}{|c|}{ Escala de 0 a 10 puntos. Siendo 0 “nula o nada adecuada” y el 10 “muy adecuada” } \\
\hline & Media & Desviación \\
\hline $\begin{array}{l}\text { 1. Formación inicial, respecto a la gestión directiva, recibida en sus estu- } \\
\text { dios previos a la actividad profesional }\end{array}$ & 3,4 & 2,7 \\
\hline $\begin{array}{l}\text { 2. Formación que reciben los actuales estudiantes de profesorado respecto } \\
\text { a la gestión directiva }\end{array}$ & 3,3 & 2,2 \\
\hline $\begin{array}{l}\text { 3. Formación permanente que reciben directores y directoras por parte de } \\
\text { las administraciones educativas }\end{array}$ & 4,8 & 2,5 \\
\hline
\end{tabular}

\subsection{La formación permanente de los responsables de la dirección de los centros}

Los responsables de la dirección valoran en una escala de 0 a 10 puntos (siendo 0 "ninguna importancia" y 10 "mucha importancia") los temas siguientes en relación con su formación permanente para sus tareas directivas: "normativa", 8,5 puntos; "liderazgo", 8,7; "organización de centros", 8,9; 
“pedagogía-psicología”, 8,5; "evaluación de centros”, 8,2 y "nuevas tecnologías", 8,3.

TABLA 3

Formación permanente de los responsables de la dirección de los centros

\begin{tabular}{|c|c|c|c|c|}
\hline \multicolumn{5}{|c|}{ Escala de 0 a 10 puntos. Siendo 0 "nula o nada adecuada" y el 10 "muy adecuada } \\
\hline \multicolumn{5}{|c|}{ 1. Normativa } \\
\hline & Total & Directoras & Directores & Grado de significación \\
\hline Media & 8,5 & 9,0 & 8,0 & \multirow{2}{*}{0,014} \\
\hline Desviación & 1,7 & 1,1 & 2,1 & \\
\hline \multicolumn{5}{|c|}{ 2. Liderazgo } \\
\hline & Total & Directoras & Directores & Grado de significación \\
\hline Media & 8,7 & 8,9 & 8,5 & \multirow{2}{*}{-} \\
\hline Desviación & 1,6 & 1,1 & 2,1 & \\
\hline \multicolumn{5}{|c|}{ 3. Organización de centros } \\
\hline & Total & Directoras & Directores & Grado de significación \\
\hline Media & 8,9 & 9,2 & 8,5 & \multirow{2}{*}{-} \\
\hline Desviación & 1,6 & 0,9 & 2,0 & \\
\hline \multicolumn{5}{|c|}{ 4. Pedagogía-psicología } \\
\hline & Total & Directoras & Directores & Grado de significación \\
\hline Media & 8,5 & 8,7 & 8,1 & \multirow{2}{*}{-} \\
\hline Desviación & 1,7 & 1,2 & 2,0 & \\
\hline \multicolumn{5}{|c|}{ 5. Evaluación de centros } \\
\hline & Total & Directoras & Directores & Grado de significación \\
\hline Media & 8,2 & 8,5 & 7,8 & \multirow{2}{*}{-} \\
\hline Desviación & 1,8 & 1,2 & 2,2 & \\
\hline \multicolumn{5}{|c|}{ 6. Nuevas tecnologías } \\
\hline & Total & Directoras & Directores & Grado de significación \\
\hline Media & 8,3 & 8,9 & 7,5 & \multirow{2}{*}{.002} \\
\hline Desviación & 1,8 & 1,2 & 2,2 & \\
\hline
\end{tabular}




\subsection{Satisfacción y reconocimiento del cargo de director o directora}

Directores y directoras valoran con 7,9 puntos su satisfacción en el cargo, en una escala de 0 a 10 puntos (siendo el valor 0 "ninguna satisfacción" y el 10 la "máxima satisfacción").

Otorgan 8,7 puntos (en la escala de 0 a 10, siendo el valor 0 "ninguna importancia" y el 10 la "máxima importancia") a la relevancia de dicho cargo para el buen funcionamiento del centro, y con 7,9 a la importancia para la calidad de la enseñanza que ofrece el centro.

El apoyo y la colaboración que tienen por parte de las administraciones educativas lo valoran con 6,7 puntos (en la escala de 0 a 10 , siendo el valor 0 “ningún apoyo y colaboración” y el 10 el "máximo apoyo y colaboración").

El reconocimiento de su trabajo por las administraciones educativas lo valoran con 5,3 puntos (en la escala de 0 a 10, siendo el valor 0 "ningún reconocimiento" y el 10 el "máximo reconocimiento"). A dicho reconocimiento por parte del profesorado y las familias le otorgan 7,6 puntos.

\subsection{Carrera profesional, retribución económica y colaboración} con la función directiva

Se valora con 8,7 puntos (en la escala de 0 a 10 , siendo el 0 "nada de acuerdo" y el 10 "totalmente de acuerdo") el que los docentes tengan una auténtica carrera profesional.

La retribución económica que perciben por el desempeño de sus funciones directivas la valoran con 4,8 puntos (en la escala de 0 a 10 puntos, siendo el 0 "muy mal" y el 10 "muy bien").

La colaboración del profesorado con la dirección del centro se valora con 7,3 puntos (en la escala de 0 a 10, siendo 0 "muy mala" y el 10 "muy buena"), y su colaboración con el centro en general con 7,6 puntos. Observamos que dichas valoraciones están muy próximas, lo que significa que no existen diferencias importantes. La colaboración con el centro por parte de las familias recibe una puntuación media de 6,8 puntos. 


\subsection{Importancia de las funciones y competencias de la dirección para el funcionamiento de los centros y la calidad de la enseñanza que ofrecen}

Directores y directoras valoran como importantes las funciones y competencias que tienen atribuidas según la normativa vigente, en una escala de 0 a 10 puntos (siendo el 0 "nada importante" y el 10 "muy importante"). El recorrido de dichas valoraciones va del menor valor de 7,2 puntos, de "realizar contrataciones de obras, servicios y suministros", a la mayor valoración de 9,4 puntos, de "favorecer la convivencia en el centro e impulsar la colaboración con las familias".

Una puntuación de 8 a 8,9 puntos se otorga a "ostentar la representación del centro" ( 8,1 puntos), "ejercer la dirección pedagógica y promover la innovación educativa" ( 8,9 puntos), "ejercer la dirección pedagógica y promover la innovación educativa" (8,9 puntos), "impulsar las evaluaciones" (8,1 puntos), "convocar y presidir los actos académicos" ( 8,7 puntos), "proponer nombramientos" (8,5 puntos), "aprobar la programación general anual" (8,1 puntos) e "impulsar la colaboración con instituciones" (8,8 puntos).

Tiene una valoración de 9,1 puntos "garantizar el cumplimiento de las leyes", y de 9,1 puntos "fomentar un clima escolar que favorezca el estudio".

No se encuentran diferencias significativas al aplicar las pruebas de contraste de medias y no paramétrica de la $U$ de Mann-Whitney respecto a las valoraciones que se realizan según las variables género, edad, experiencia (docente y como director o directora) y titularidad del centro.

\section{DisCUSIÓN Y CONCLUSIONES}

Como primer apunte, observamos que directores y directoras no están demasiado satisfechos con la formación inicial que han recibido en su etapa universitaria en relación con su actual actividad profesional, a la vez que consideran susceptible de mejora la formación permanente que les proporcionan las administraciones educativas en el ejercicio de sus funciones directivas. Valoraciones que indican que debe tenerse en cuenta lo señalado por Fernández-Serra (2002) respecto a la necesidad de que el proceso de formación 
debe comenzar antes de su nombramiento y desarrollarse durante toda su etapa directiva. Así como por González-Álvarez (2017a, 2017b), que señala que dicha formación inicial debe mejorarse y que la permanente precisa de alguna mejora y adaptación a las necesidades específicas. Aramendi, Teixidó y Bernal (2010) también consideran que la gestión directiva debe incluirse en los currículos universitarios de formación del profesorado.

Los temas objeto de formación que hemos propuesto son valorados como bastante importantes por parte de los directores y las directoras consultados. Por ello, podrían incluirse en los programas de formación tanto inicial como permanente que se entiende que deben elaborarse al efecto.

Los directores y las directoras consultados manifiestan tener una alta satisfacción en su cargo, coincidiendo en gran medida con lo señalado en el Informe TALIS (2009), en el que se indicaba dicha satisfacción para el 97,5 \% de los cargos directivos. Satisfacción en la que se incluye el adecuado apoyo y la colaboración que manifiestan tener por parte del profesorado y de las familias. Reconocimiento que consideran menor respecto al que reciben de las administraciones educativas.

De igual manera consideramos que, según valoran directores y directoras, la función que ejerce es importante para el funcionamiento del centro y para la calidad de la enseñanza que ofrecen a su alumnado. Coincidiendo con lo indicado por los autores señalados (Branch, Hanushek y Rivkin, 2012; Fernández-Serra, 2002; Gairín y Castro, 2010; Mulford, 2006; Ritacco y Bolívar, 2016; Vázquez, Liesa, y Gayán, 2013; Waters, Marzano y McNulty, 2003), respecto a considerar la función directiva de los centros educativos como un factor fundamental para su buen funcionamiento y la mejora de la enseñanza de su alumnado.

Por otro lado, los directores y las directoras consultados están muy de acuerdo con que exista una auténtica carrera profesional para los docentes a la vez que entienden que puede mejorarse la retribución económica que perciben por el desempeño de su cargo.

Directores y directoras consideran importantes para el funcionamiento del centro y la enseñanza que imparte a su alumnado las funciones y competencias que tienen asignadas por la norma.

Entendemos que, según señalan Álvarez-Fernández (2004), Gairín y Castro (2010) y Martín-Moreno (2007), la función directiva debe prestar especial 
atención a las actuales demandas sociales, por lo que procede analizar su actual situación.

Consideramos la importancia que debe darse a la formación de directores y directoras, tanto inicial como permanente.

Las administraciones educativas deberían prestar un mayor reconocimiento a los responsables de la dirección de los centros de Educación Infantil y Primaria y de manera específica en lo que se refiere a la retribución económica que deban percibir por sus tareas.

\section{BIBLIOGRAFÍA}

Álvarez-Fernández, M. (2004). Dirección y calidad de la educación. El rendimiento del centro escolar. Enseñanza, 22, 77-102. Recuperado de: https://gredos.usal.es/jspui/bitstream/.../Direccion_y_calidad_de_la_educacion_El_r.p...

Aramendi, P.; Teixidó, J. y Bernal, J. L. (2010). El acceso a la dirección escolar en los centros públicos del País Vasco. Revista Española de Pedagogía, 246, 313-332. Recuperado de: https://dialnet.unirioja.es/descarga/ articulo/3206706.pdf.

Barrios-Arós, C. H.; Camarero-Figuerola, M.; Tierno-García, J. M. e Iranzo-García, P. (2015). Modelos y funciones de dirección escolar en España: El caso de Tarragona. Revista Iberoamericana de Educación, 67, 89106. Recuperado de: https://rieoei.org/historico/documentos/rie67a05.pdf.

Batanaz, L. (2005). El acceso a la dirección escolar. Problemas y propuestas. Revista Española de Pedagogía, 63(232), 443-470. Recuperado de: https:/dialnet.unirioja.es/descarga/articulo/1373228.pdf.

Bolívar, A. y Cerrillo, R. (2015). Evaluación del liderazgo escolar. Revista Iberoamericana de Educación, 8(2), 11-14. Recuperado de: http://www. rinace.net/riee/numeros/vol8-num2.html.

Bolívar, A. y Ritacco, M. (2017). Una doble y discontinua identidad profesional: Los directores escolares en España en una dimensión biográfico narrativa. En B. Merrill, J. González-Monteagudo, A. Nizinska, A. Galimberti y, M. A. Ballesteros-Moscosio (eds), Adult Learning, Educational Careers and Social Change (pp. 12-25). Sevilla: Universidad de Sevilla/ 
ESREA. Recuperado de: https://revistas.cardenalcisneros.es/index.php/ PULSO/article/view/307.

Branch, G. F.; Hanusher, E. A. y Rivin, S. G. (2012). Estimating the effect of leaders on public sector productivity: the case of school principals. National Bureau of Economic Research. Recuperado de: https://files.eric .ed.gov/fulltext/ED529175.pdf.

BRASLAVSK, C. (2006). Diez factores para una educación de calidad para todos en el siglo xxi. Revista Electrónica Iberoamericana sobre Calidad, Eficacia y Cambio en Educación, 4(2e), 84-101. Recuperado de: www.rinace. net/arts/vol4num2e/art5.pdf.

Caballero, J. y Mata, F. S. (2004). Satisfacción e insatisfacción de los directores escolares. Revista de Educación, 233, 363-384. Recuperado de: https://dialnet.unirioja.es/servlet/articulo? codigo $=856269$.

Calatayud, M. A. (2015). El liderazgo emergente de los directores escolares en España. La voz del profesorado. Revista Iberoamericana de Educación, 69, 207-228. Recuperado de: https://rieoei.org/historico/documentos/rie69a09.pdf.

Educobarómetro (2010). La opinión de los equipos directivos. Instituto de Evaluación y Asesoramiento Educativo. Fundación SM.

Escobar-Pérez, A. y Cuervo-Martínez, J. (2008). Validez de contenido y juicio de expertos una aproximación a su utilización. Avances en Medición, 6, 27-36. Recuperado de: http://www.humanas.unal.edu.co/psicometria/ files/7113/8574/5708/Articulo3 juicio_de_expertos_27-36.pdf.

Escudero-Muñoz, J. M.; GonZÁlez-GonZÁlez, M.T. y RodríGuez-EnTRENA, M. J. (2018). Los contenidos de la formación continuada del profesorado: ¿Qué docentes se están formando? Educación XX1, 21(1), 157180. Recuperado de: https://www.researchgate.net/.../320905669_LOS_ CONTENIDOS_DE_LA_FORMACIO...

FernándeZ-SerRA, M. L. (2002). Formar para la dirección escolar: Por qué, cuándo, cómo... Revista de Currículum y Formación del Profesorado, 6(12). Recuperado de: http://www.ugr.es/ recfpro/rev61COL2.pdf.

Gairín, J. y CASTRO, D. (2010). Situación actual de la dirección y gestión de los centros de enseñanza obligatoria en España. Revista Española de Pedagogía, 247, 401-416. Recuperado de: https://dialnet.unirioja.es/descarga/articulo/3300518.pdf. 
García, A.; Poblete, M. y Villa, A. (2006). La función directiva: un problema sin resolver. Tres décadas de formación, investigación y acción. XXI Revista de Educación, 8, 13-34. Recuperado de: https://rabida.uhu.es/dspace/handle/10272/2011.

GarcíA-GARnicA, M. (2016). La relevancia del liderazgo pedagógico para la mejora y la calidad de la educación. Revista Internacional de Didáctica y Organización Educativa, 1(2), 4-20. Recuperado de: http://www.redoe. com/index.php?journal=reido.

Gómez-Delgado, A. M.; Oliva-Rodríguez, N.; LóPez-YÁñez, J. (2014). La indefinición del modelo español de formación de líderes escolares. REICE. Revista Iberoamericana sobre Calidad, Eficacia y Cambio en Educación, 12(5), 135-150. Recuperado de: www.redalyc.org/ pdf $/ 551 / 55132460006$.

GonzÁlez-Álvarez, M. (2017a). Formación inicial y permanente de directivos de Infantil y Primaria. Magisterio Español, 12.131, 15-17.

GonzÁlez-Álvarez, M. (2017b). La dirección de centros de Infantil y Primaria de Cataluña. Magisterio Español, 12.134, 15-16.

Grissom, J. A. y LoEB, S. (2011). Triangulating principal effectiveness: How perspectives of parents, teachers, and assistant principals identify the central importance of managerial skills. American Education Research Journal, 48(5), 1091-1123. Recuperado de: https://www.urban.org/.../1001443Triangulating-Principal-Effect...

Hess, F. M. y Kelly, A. P. (2007). Learning to lead: What gets taught in principal-preparation programs. Teachers College Record, 109(1), 221-243. Recuperado de: https://sites.hks.harvard.edu/.../Hess_Kelly_Learning_to_ Lead_PE...

InSTITUTO DE EVALUACión TALIS (OCDE) (2009). Estudio internacional sobre la Enseñanza y el Aprendizaje. Informe español 2009. Madrid: Ministerio de Educación.

Martín-Moreno, Q. (2007). Desafíos persistentes y emergentes para las organizaciones educativas. Bordón Revista de Pedagogía, 59(2-3), 417-430. Recuperado de: https://dialnet.unirioja.es/ejemplar/184892.

Mulford, B. (2006). Liderazgo para mejorar la calidad de la educación secundaria: algunos desarrollos internacionales. Profesorado. Revista de cu- 
rrículum y formación del profesorado, 10(1). Recuperado de: https://recyt. fecyt.es/index.php/profesorado/article/view/42024.

Murillo, F. J. y Hernández-Castilla, R. (2015). Liderazgo para el aprendizaje: ¿Qué tareas de los directores y directoras escolares son las que más inciden en el aprendizaje de los estudiantes? Relieve, 21(1), art. 1. Recuperado de: https://ojs.uv.es/index.php/RELIEVE/article/view/501.

Ritacco, M. y Bolívar, A. (2016). Impacto del modelo español de dirección escolar en la identidad profesional de los líderes escolares. Archivos Analíticos de Políticas Educativas, 24(119). Recuperado de: http://dx.doi. org/10.14507/epaa.24.2512.

Tejero, C. M. y Fernández, M. J. (2009). Medición de la satisfacción laboral en la dirección escolar. RELIEVE. Revista Electrónica de Investigación y Evaluación Educativa, 15(2). Recuperado de: www.redalyc.org/articulo. oa?id=91612906002.

Tellez, S. (2015). La satisfacción de los profesores de los centros educativos de educación infantil y educación primaria en las comarcas del Bierzo y Valdeorras. León: Universidad de León (tesis doctoral). Recuperado de: https://buleria.unileon.es/handle/10612/599.

VÁzquez, S.; Liesa, M. y Bernal, J. L. (2015). Análisis de la formación en liderazgo en las organizaciones educativas: un estudio en la Comunidad Autónoma de Aragón. Revista Electrónica Interuniversitaria de Formación del Profesorado, 18(3), 39-54. Recuperado de: revistas.um.es/reifop/ article/download/194881/181781.

VÁzQUez, S.; Liesa, M. y GAYÁn, R. (2013). Formación para la función directiva en Aragón. Forum Aragón, 9, 34-37. Recuperado de: https://dialnet. unirioja.es/descarga/articulo/4335682.pdf.

Waters, T.; Marzano, R. y Mcnulty, B. (2003). Balanced leadership: What 30 years of research tells us about the effect of leadership on student achievement. Aurora, CO: Mid-Continent Research for Education and Learning. Recuperado de: https://eric.ed.gov/?id=ED481972. 